\title{
Randomized phase Il study of anastrozole plus tegafur- uracil as neoadjuvant therapy for ER-positive breast cancer in postmenopausal Japanese women (Neo-ACET BC)
}

\author{
Takahiro Nakayama ${ }^{1}$ - Yasuaki Sagara ${ }^{2} \cdot$ Tsutomu Takashima $^{3} \cdot$ Nobuki Matsunami $^{4} \cdot$ Norikazu Masuda $^{5}$. \\ Yasuo Miyoshi ${ }^{6} \cdot$ Tetsuya Taguchi $^{7} \cdot$ Toyokazu Aono $^{8} \cdot$ Toshikazu $_{\text {Ito }}{ }^{9} \cdot$ Tatsuo Kagimura $^{10} \cdot$ Shinzaburo Noguchi $^{11}$
}

Received: 16 January 2018 / Accepted: 12 February 2018 / Published online: 21 February 2018

(c) The Author(s) 2018. This article is an open access publication

\begin{abstract}
Purpose This phase II study evaluated the efficacy and safety of anastrozole concurrent with tegafur/uracil (UFT) as neoadjuvant therapy for ER-positive postmenopausal breast cancer.

Methods Postmenopausal Japanese women with ER-positive, HER2-negative, T2,N0-1,M0 breast cancer seen at tertiary hospitals were eligible for this open-label, randomized, multicenter study. Patients were randomized 1:1 by minimization to orally receive either anastrozole ( $1 \mathrm{mg}$ once daily) plus UFT (tegafur/uracil combination in 1:4 molar ratio; $270 \mathrm{mg} / \mathrm{m}^{2} /$ day in two divided doses) or anastrozole (as above) alone for 24 weeks. Tumor response was assessed by investigator and central review as per RECIST v1.1. The primary endpoint was the proportion of patients with best overall response of CR or PR [clinical response rate (RR)] determined by central radiologic review.

Results The study was prematurely terminated due to Grade $\geq 3$ liver dysfunction reported in 3 patients receiving anastrozole/ UFT. Of 57 patients randomized before termination ( 29 anastrozole/UFT, 28 anastrozole), all were analyzed for safety and 56 (28 each group) for tumor response. Compared with anastrozole alone, anastrozole/UFT did not achieve significantly higher RR [39.3\% (90\% CI 23.8-56.5\%) vs 14.3\% (90\% CI 5.0-29.8\%); $p=0.0683$, Fisher's exact test], but produced significantly greater tumor shrinkage (mean tumor reduction rate 31.0 vs. $14.2 \% ; p=0.0181$, unpaired $t$-test). Grade $\geq 3$ adverse events were more common with anastrozole/UFT than with anastrozole (17.2 vs. $0 \%)$.

Conclusion Although the study was terminated owing to the altered liver function, it showed that there was a trend to greater shrinkage of tumor in the combination group for ER-positive, HER2-negative postmenopausal breast cancer.
\end{abstract}

Keywords Breast cancer $\cdot$ Anastrozole $\cdot$ UFT $\cdot$ Clinical study $\cdot$ Neoadjuvant therapy

Takahiro Nakayama

taqnakayama@gmail.com

1 Department of Breast and Endocrine Surgery, Osaka International Cancer Institute, 3-1-69, Otemae, Chuou, Osaka 541-8567, Japan

2 Department of Breast Surgery, Sagara Hospital, Kagoshima, Japan

3 Department of Surgical Oncology, Osaka City University Hospital, Osaka, Japan

4 Department of Surgery, Osaka Rosai Hospital, Sakai, Japan

5 Department of Surgery, Breast Oncology, National Hospital Organization Osaka National Hospital, Osaka, Japan
6 Department of Surgery, Division of Breast and Endocrine Surgery, Hyogo College of Medicine, Nishinomiya, Japan

7 Department of Endocrine and Breast Surgery, Kyoto Prefectural University of Medicine, Kyoto, Japan

8 Department of Breast Surgery, Osaka Breast Clinic, Osaka, Japan

9 Department of Surgery, Rinku General Medical Center, Izumisano, Japan

10 Translational Research Informatics Center, Foundation for Biomedical Research and Innovation, Kobe, Japan

11 Department of Breast and Endocrine Surgery, Graduate School of Medicine Osaka University, Suita, Japan 


\section{Introduction}

Breast cancer, even at its early stage, often presents with disseminated micrometastases, which cannot be adequately controlled by locoregional treatments based on surgery and radiotherapy. Neoadjuvant and adjuvant systemic chemotherapy and endocrine therapy have been proposed to eradicate these micrometastases [1-3]. Neoadjuvant chemotherapy, which has been the mainstay of neoadjuvant medical breast cancer treatment, has proven benefits, such as increasing the likelihood of breast conservation and improving the longterm prognosis in patients achieving pathological complete response (pCR). Of note, some investigators have reported similar long-term prognosis of breast cancer patients after neoadjuvant chemotherapy to that after adjuvant (postoperative) chemotherapy [4]. Factors known to affect the breast cancer response to neoadjuvant chemotherapy include hormone-receptor (HR) status and human epidermal growth factor receptor 2 (HER2) status of the tumor. In patients with estrogen receptor (ER)-positive, HER2-negative tumors, neoadjuvant chemotherapy has limited activity, rarely causing $\mathrm{pCR}$, and has not been shown to improve the long-term prognosis $[5,6]$. Neoadjuvant endocrine therapy with an aromatase inhibitor (AI) has emerged as a newer strategy for breast cancer of this phenotype [7]. A randomized controlled study has shown comparable efficacy of neoadjuvant AI versus chemotherapy for ER-positive, HER2-negative, postmenopausal breast cancer with regard to $\mathrm{pCR}$ and breast conservation rates, but significantly less adverse events with AI than with chemotherapy [8]. However, breast cancers with aggressive clinical features such as Luminal B subtype are less responsive to endocrine treatments, showing poor response even to neoadjuvant AI. Regarding cytotoxic agents, metronomic dosing has been devised to avoid their dose-dependent toxicities and to sustain their effects, so as to produce antiangiogenesis that can indirectly lead to an inhibition of tumor cell proliferation. Metronomic therapy with cyclophosphamide and UFT (tegafur/uracil combination in a fixed molar ratio of 1:4) has gained wide acceptance $[9,10]$. Anastrozole administered in combination with UFT exhibited significantly higher antitumor activity as compared with either agent alone against a human breast cancer cell line grown in mice [11], suggesting promising activity of endocrine therapy concurrent with metronomic chemotherapy for breast cancer in humans.

With these backgrounds, we conducted this randomized phase II study to evaluate anastrozole plus UFT as neoadjuvant therapy for HR-positive breast cancer in postmenopausal Japanese women.

\section{Materials and methods}

\section{Study design}

This was an open-label, randomized, multicenter, phase II study designed to evaluate the efficacy and safety of anastrozole plus UFT versus anastrozole alone as neoadjuvant therapy for ER-positive, HER2-negative breast cancer in postmenopausal Japanese women. The study was approved by the institutional review board of each study site and was conducted in accordance with the Declaration of Helsinki and ethical principles of clinical investigations. Prior to enrollment, all patients provided informed consent to the study. The study has been registered with UMIN-CTR (UMIN000006434).

\section{Patients and treatment}

Eligible patients were postmenopausal women with ERpositive ( $\geq 10 \%)$, HER2-negative (immunohistochemistry staining score 0 or $1+$, or fluorescence in situ hybridization negative), T2,N0-1,M0 invasive ductal or lobular carcinoma of breast who had an Eastern Cooperative Oncology Group (ECOG) performance status (PS) score of 0 or 1 . Patients were centrally enrolled and randomized 1:1 to receive anastrozole plus UFT or anastrozole alone. Randomization was done by the minimization method following stratification with respect to extent of lymph node involvement (N0 or N1) and progesterone receptor (PgR) status of the tumor (positive, negative or unknown).

Patients allocated to the anastrozole alone group were given oral anastrozole at $1 \mathrm{mg}$ once daily for 24 weeks, whereas those allocated to the anastrozole plus UFT group were given oral anastrozole at the same dosage in addition to oral UFT at $270 \mathrm{mg} / \mathrm{m}^{2} /$ day (recommended daily dose per body surface area at enrollment) in two divided doses for 24 weeks. Throughout the treatment period, patients in both groups were prohibited to receive any non-study treatment for breast cancer (e.g., hormones other than anastrozole, cytotoxic agents, radiotherapy, and biological response modifiers). Patients in the anastrozole/UFT group were also prohibited to receive any antineoplastic agent other than UFT. All patients were to undergo surgery at 1-4 weeks after the end of the protocol neoadjuvant treatment.

\section{Endpoints and statistical analysis}

The primary endpoint was the proportion of patients with best overall response of CR or PR (clinical response rate [RR]) determined by central radiologic review as per RECIST v1.1. A tumor lesion with larger diameter $\geq 10 \mathrm{~mm}$ 
as measured by MRI (preferred) or CT and $\geq 10 \mathrm{~mm}$ as measured using a caliper was defined as measurable. Tumor size was measured by MRI or CT at the start and the end of the protocol treatment and using a caliper and ultrasonography every 4 weeks during the treatment. Overall response was determined every 4 weeks based on combined assessment of the response of target lesion(s) and appearance of any new lesion, and the best of the overall responses seen during the treatment period was defined as best overall response. Secondary endpoints included percent changes in tumor diameter measured by MRI or CT from baseline to the end of treatment for individual patients (tumor reduction rate), histological tumor response, and proportion of patients undergoing breast conserving surgery (breast conservation rate) as well as severity and frequency of adverse events (AEs). Histological tumor response was assessed according to criteria adapted from the General Rules for Clinical and Pathological Recording of Breast Cancer ver16 (2008), and the proportions of patients showing Grade $\geq 1 \mathrm{~b}$ response was calculated. AEs were coded and graded using the CTCAE v4.0.

By reference to RR values previously obtained with neoadjuvant anastrozole using caliper-based tumor size measurements $[12,13]$, the threshold RR was assumed to be $45 \%$. The least number of subjects needed to show an expected RR of $65 \%$ for anastrozole plus UFT with a one-sided alpha error rate of 5\% and a statistical power of $90 \%$ was calculated as 53. Hence, a sample size of 120 (60 per group) was planned by taking into account the potential of excluding ineligible subjects.

The efficacy analysis set was composed of all randomized patients but those who proved to be ineligible after randomization. The safety analysis set was composed of all randomized patients but those who received no doses of the protocol treatment assigned.

For RR (primary endpoint), the $90 \%$ confidence interval (CI) was calculated to test whether the lower bound of the $90 \%$ CI for the RR obtained with anastrozole plus UFT was at or above the threshold RR (45\%). In an exploratory manner, RR data were compared between the two groups using Fisher's exact test. Among secondary endpoints, tumor reduction rates were compared between the two groups using the unpaired $t$-test. All tests were two-sided, and a $p$ value less than 0.05 was considered statistically significant.

\section{Results}

Accrual was suspended on March 27, 2012, when 57 patients had been enrolled because of serious liver dysfunction [increased alanine aminotransferase (ALT) and increased aspartate aminotransferase (AST)] reported in 3 of 29 patients assigned to the anastrozole plus UFT group by this date. Subsequently, the data center performed an interim analysis of data from the 57 patients. The independent data monitoring board concluded that the results of the analysis did not warrant continuation of the study and recommended the protocol committee to terminate the study. Following the recommendation, the committee decided to terminate the study on February 19, 2014.

\section{Patient characteristics}

All 57 patients enrolled between December 1, 2010 and March 27, 2012 were randomized to receive either anastrozole plus UFT $(n=29)$ or anastrozole alone $(n=28)$. All of them were evaluated for safety, while 28 in each group were evaluated for tumor response (Fig. 1). The patient randomized to the anastrozole plus UFT group and excluded from the efficacy analysis set proved to be ineligible after randomization. The two treatment groups were well-matched with respect to baseline characteristics (Table 1).

\section{Efficacy}

As shown in Table 2, the anastrozole plus UFT group had RR (90\% CI) of 39.3\% (23.8-56.5\%) with two CRs (7.1\%) and 9 PRs (32.1\%); the lower bound of the $90 \% \mathrm{CI}$ was less than the threshold RR (45\%). Thirteen patients (46.4\%) had best overall response of SD. The RR in the experimental group was numerically but not significantly higher than that in the control group [14.3\% (90\% CI 5.0-29.8\%); $p=0.0683$, Fisher's exact test]. In the experimental and control groups, two versus zero patients achieved CR, while no versus 3 patients experienced PD. Of 25 and 27 patients evaluable for histological tumor response in the respective groups, 8 (32.0\%) and 12 (44.4\%) patients showed Grade $\geq 1 b$ histological response as per the criteria adapted from the General Rules for Clinical and Pathological Recording of Breast Cancer ver16.

Figure 2 shows a waterfall plot of tumor reduction rate. As compared with anastrozole alone, anastrozole plus UFT produced significantly greater tumor shrinkage with the

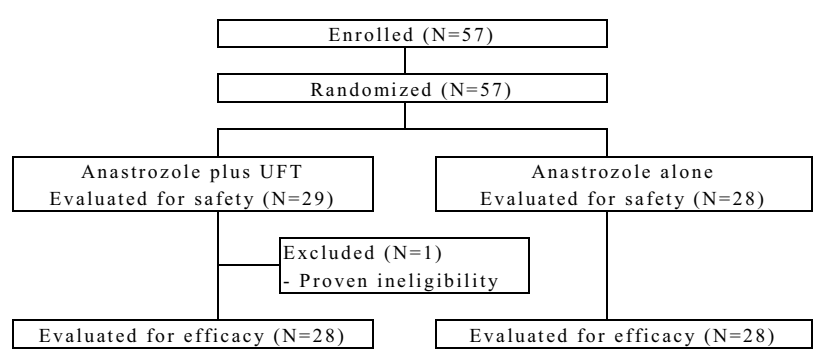

Fig. 1 Subject disposition 
Table 1 Patient characteristics

\begin{tabular}{lccc}
\hline$N(\%)$ & $\begin{array}{c}\text { Anastrozole plus } \\
\text { UFT }(N=29)\end{array}$ & $\begin{array}{l}\text { Anastrozole alone } \\
(N=28)\end{array}$ & $P$ value* \\
\hline Age [years], median (range) & $70(51-87)$ & $65.5(50-81)$ & 0.0901 \\
BSA $\left[\mathrm{m}^{2}\right]$, median (range) & $1.5(1.3-1.9)$ & $1.5(1.3-1.9)$ & 0.0935 \\
$N$ status & & & 1.0000 \\
N0 & $21(72.4)$ & $20(71.4)$ & \\
N1 & $8(27.6)$ & $8(28.6)$ & 1.0000 \\
PgR-IHC & & & \\
Negative & $8(27.6)$ & $20(71.4)$ & 1.0000 \\
Positive & $21(72.4)$ & $26(92.9)$ & \\
Histological subtypes & & $2(7.1)$ & \\
Invasive carcinoma of no special type & $27(93.1)$ & & \\
Special subtypes (invasive lobular carcinoma) & $2(6.9)$ & $19(67.9)$ & \\
Form of surgery offered at presentation & $15(51.7)$ & $9(32.1)$ & \\
Breast conserving surgery & $14(48.3)$ & $26(92.9)$ & \\
Mastectomy & & $2(7.1)$ & \\
PS (ECOG) & $27(93.1)$ & $2(6.9)$ & \\
0 &
\end{tabular}

$B S A$ body surface area, ECOG Eastern Cooperative oncology group, $I H C$ immunohistochemistry, $P g R$ progesterone receptor, $P S$ performance status

*Wilcoxon rank sum test or Fisher's exact test

Table 2 Clinical response rate (RR)

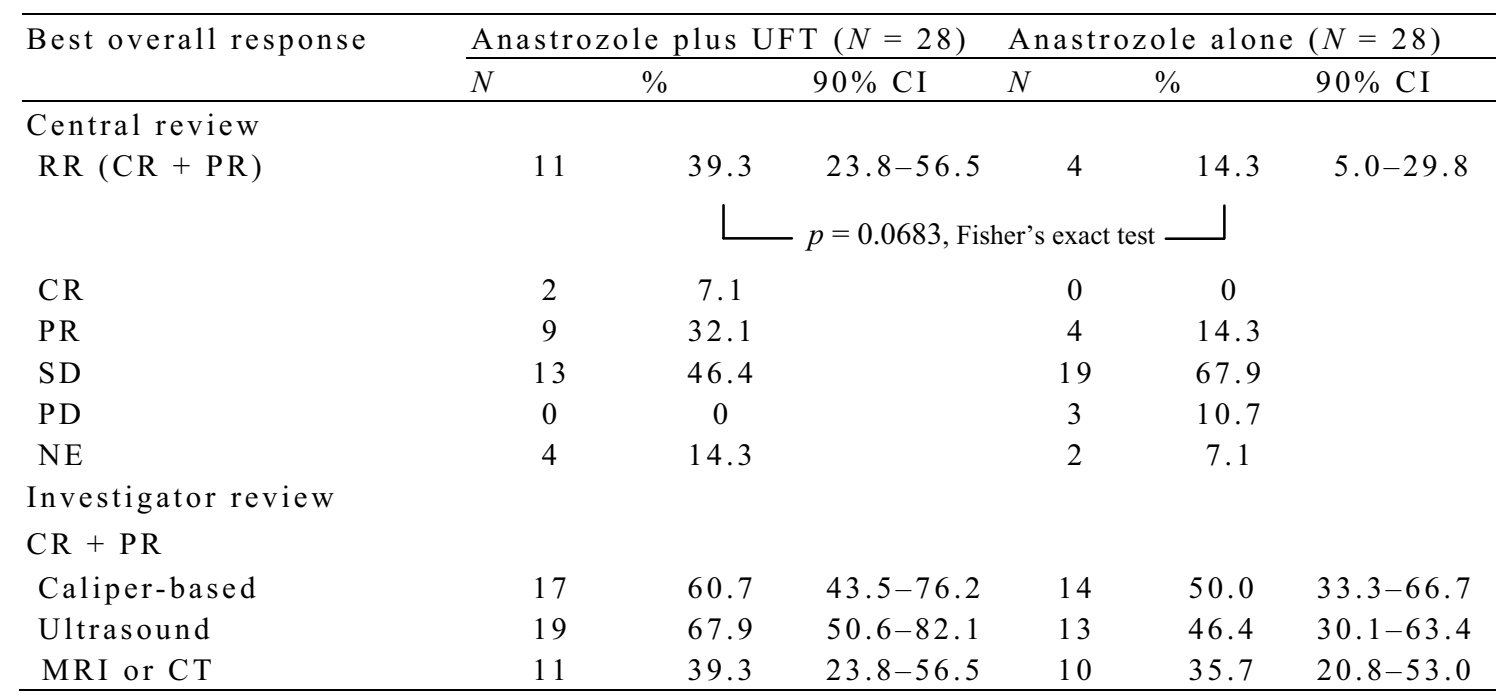

mean (SD) tumor reduction rate being 31.0 (29.17) \% versus $14.2(17.30) \%$ ( $p=0.0181$, unpaired $t$-test).

After receiving the protocol treatment, 25 patients in the anastrozole plus UFT group and 28 in the anastrozole alone group were offered surgery. Of these, 19 [76.0\% (95\% CI 54.9-90.6\%)] and 16 [57.1\% (95\% CI $37.2-75.5 \%)$ ] patients underwent breast conserving surgery.

\section{Safety}

AEs occurred in 22/29 patients (75.9\%) in the anastrozole plus UFT group versus $17 / 28$ patients $(60.7 \%)$ in the anastrozole alone group ( $p=0.2630$, Fisher's exact test). Grade $\geq 3$ AEs were more frequent in the anastrozole plus UFT group $(n=5,17.2 \%)$ than in the anastrozole alone group $(n=0$, $0 \%)(p=0.0518$, Fisher's exact test). The most common AEs 
Fig. 2 Waterfall plot of tumor reduction rate (percent reduction in unidimensional radiologic measurements)

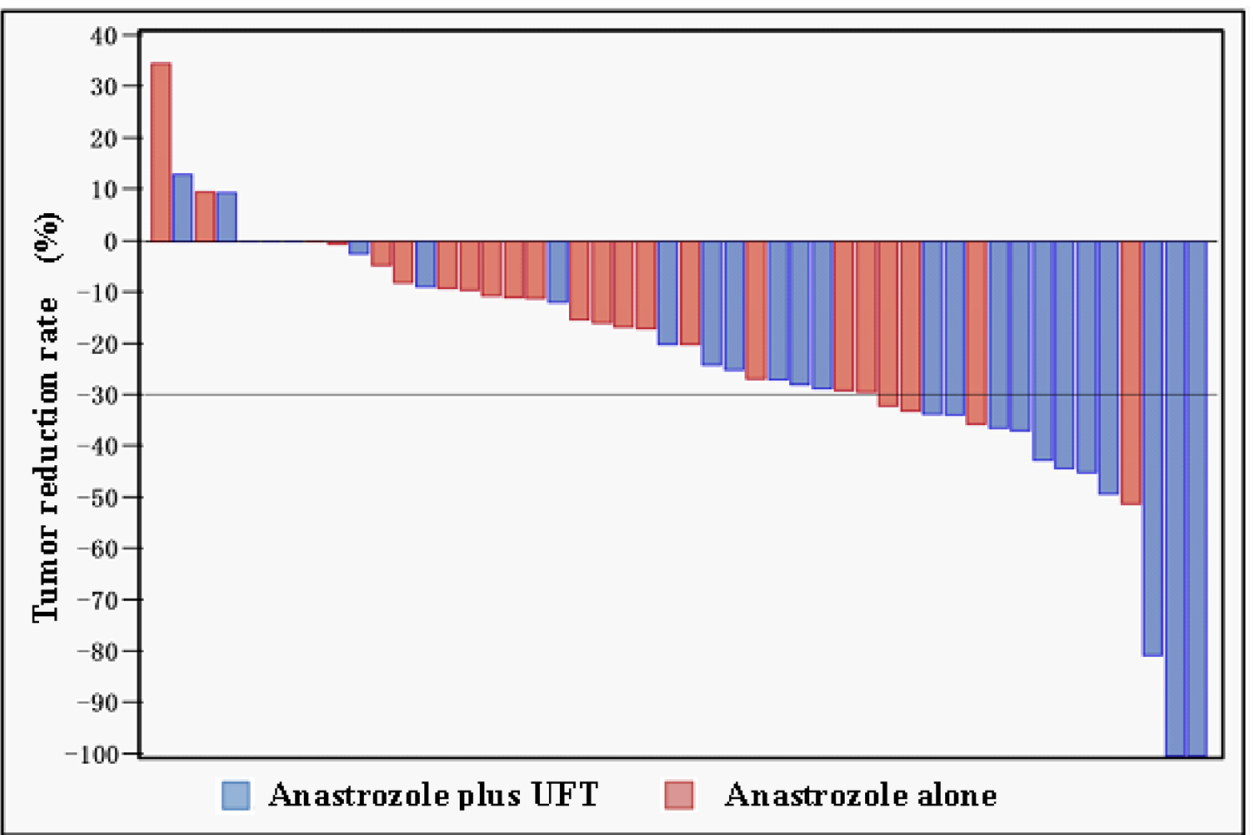

Tumor reduction rate as per investigator assessment

\begin{tabular}{ccc}
\hline & \multicolumn{2}{c}{ Treatment groups } \\
\cline { 2 - 3 } & $\begin{array}{c}\text { Anastrozole plus UFT } \\
(\mathbf{N}=\mathbf{2 8})\end{array}$ & $\begin{array}{c}\text { Anastrozole alone } \\
(\mathbf{N}=\mathbf{2 8})\end{array}$ \\
\hline Pts assessed, $N$ & 25 & 24 \\
Mean & 31.0 & 14.2 \\
Standard deviation & 29.17 & 17.30 \\
Minimum & -13.0 & -34.5 \\
Median & 27.80 & 13.15 \\
Maximum & 100.0 & 51.1 \\
\hline Pts with missing data, $N$ & 3 & 4 \\
\hline$p t s$ patients & & Unpaired $t$-test, $p=0.0181$
\end{tabular}

in the anastrozole plus UFT group included increased ALT (55.2\%), increased AST (48.3\%), increased blood bilirubin $(41.4 \%)$, thrombocytopenia $(31.0 \%)$, and fatigue $(31.0 \%)$ (Table 3). Grade 3 AEs were increased AST (10.3\%), increased ALT (10.3\%), back pain (3.4\%), and hypertriglyceridemia (3.4\%). No Grade $\geq 3$ AEs were reported in the anastrozole alone group. No deaths occurred in either group.

\section{Discussion}

In this randomized phase II study, we evaluated the efficacy and safety of anastrozole plus UFT versus anastrozole alone as neoadjuvant therapy for ER-positive, HER2-negative postmenopausal breast cancer.

Although the study was terminated owing to the altered liver function, it showed there was a trend to greater shrinkage of tumor in the combination group.

In general, tumor cells with higher proliferative activity are more sensitive to chemotherapy. Endocrine therapy inhibits the proliferation of tumor cells and therefore counteracts the cytotoxic activity of chemotherapy used concomitantly [14]. In contrast, metronomic chemotherapy has been suggested to enhance the antitumor activity of endocrine therapy, as it inhibits tumor growth through antiangiogenesis. Previous studies have demonstrated a benefit of 2-year postoperative adjuvant therapy with UFT concurrent with tamoxifen for ER-positive breast cancer [15-18]. In the present study, anastrozole plus UFT produced a higher tumor reduction rate as compared with anastrozole alone.

During this combination therapy, 2 patients achieved CR and no patients had best overall response of PD. The former finding suggests that the addition of UFT may enhance the activity of endocrine therapy, while the latter finding reflects the activity of UFT against hormone-resistant tumor cells potentially existing in an HR-positive breast tumor. Thus, the combination of UFT with anastrozole may have conferred broad-spectrum antitumor activity that could cover hormone-sensitive as well as hormone-resistant tumors. 
Table 3 Adverse events collected in a solicited manner

\begin{tabular}{|c|c|c|c|c|}
\hline \multirow[t]{2}{*}{ Events, $N(\%)$} & \multicolumn{2}{|c|}{ Anastrozole plus UFT $(N=29)$} & \multicolumn{2}{|c|}{ Anastrozole alone $(N=28)$} \\
\hline & Any grade & Grade $\geq 3$ & Any grade & Grade $\geq 3$ \\
\hline Alanine aminotransferase increased & $16(55.2)$ & $3(10.3)$ & $6(21.4)$ & 0 \\
\hline Aspartate aminotransferase increased & $14(48.3)$ & $3(10.3)$ & $4(14.3)$ & 0 \\
\hline Blood bilirubin increased & $12(41.4)$ & 0 & 0 & 0 \\
\hline Thrombocytopenia & $9(31.0)$ & 0 & $1(3.6)$ & 0 \\
\hline Fatigue & $9(31.0)$ & 0 & $2(7.2)$ & 0 \\
\hline Anorexia & $7(24.1)$ & 0 & 0 & 0 \\
\hline Nausea & $7(24.1)$ & 0 & 0 & 0 \\
\hline Leukopenia & $5(17.2)$ & 0 & $4(14.3)$ & 0 \\
\hline Diarrhea & $4(13.8)$ & 0 & $4(14.3)$ & 0 \\
\hline Creatinine increased & $3(10.3)$ & 0 & $3(10.7)$ & 0 \\
\hline Skin hyperpigmentation & $3(10.3)$ & - & 0 & - \\
\hline Hemoglobin increased & $2(6.9)$ & 0 & $1(3.6)$ & 0 \\
\hline Mucositis oral & $2(6.9)$ & 0 & $2(7.2)$ & 0 \\
\hline Vomiting & $1(3.4)$ & 0 & 0 & 0 \\
\hline Rash maculopapular & $1(3.4)$ & 0 & $1(3.6)$ & 0 \\
\hline
\end{tabular}

This study failed to show that the lower bound of the 90\% CI for the RR in the anastrozole plus UFT group was at or above the threshold RR (45\%). We used radiological (MRI or CT) tumor measurements to determine the RR (primary endpoint) despite the use of previous response data obtained by caliper-based measurements to set the threshold RR. Radiological response assessments are generally worse than caliper-based assessments, as was observed in the present study [RR $37.5 \%(21 / 56)$ vs $55.4 \%(31 / 56)$ as per investigator assessment]. This might partially account for the failure of the study to meet its primary endpoint. The RR determined by caliper-based measurements in patients treated with anastrozole plus UFT was $60.7 \%$ (17/28), which is comparable to the expected value $(65 \%)$ and deserves evaluation.

While anastrozole plus UFT tended to produce greater clinical tumor response as compared with anastrozole alone, histological tumor response showed an opposite tendency. This might be partly due to a great inter-site, inter-rater variability in ratings of histological tumor response to neoadjuvant endocrine therapy, which is likely to occur when many of the pathologists involved have little experience in this rating.

Grade $\geq 3$ liver dysfunction occurred in 3 patients $(10.3 \%)$ in the anastrozole plus UFT group. All events resolved and none of them resulted in death. Although a previous special drug-use survey reported similar frequency of liver dysfunction between patients treated with UFT plus anastrozole and those treated with UFT alone [19], the occurrence of severe liver dysfunction after concurrent treatment with anastrozole and UFT in the present study indicates the need for careful monitoring of liver function during this combination therapy in practice.
This study had several limitations. First, the study was probably underpowered, because it was terminated before achieving the planned sample size $(n=120)$. Second, no patients enrolled in this study were followed up for survival after years, because the association of achievement of pCR after neoadjuvant treatment with a better long-term outcome remained controversial. Third, this study was conducted with no regard to classification by risk factors; luminal breast cancer with high-risk features (e.g., with a high Ki67 index) may be sensitive to chemotherapy.

Furthermore, the optimal duration of neoadjuvant therapy for breast cancer has not been established. In the present study, patients were to receive neoadjuvant endocrine or chemoendocrine therapy for 24 weeks ( 6 months). Previous studies have reported a higher breast conservation rate after 6-month versus 4-month neoadjuvant therapy [20], achievement of maximal tumor response after 6- to 12-month neoadjuvant therapy [21], and a significantly higher tumor response rate after 12 months than a shorter period of neoadjuvant therapy [22]. In the N-SAS BC06 study, the majority of postmenopausal Japanese women with breast cancer scheduled to undergo mastectomy could undergo breast conserving surgery after receiving neoadjuvant therapy for 6 months [23]. To increase the likelihood of breast conservation, neoadjuvant therapy should be continued for at least 3 to 4 months, preferably for up to 6 months, as long as the patient shows no disease progression during the therapy.

Recently, a cyclin-dependent kinase (CKD) 4/6 inhibitor has emerged as a promising new drug for HR-positive breast cancer. The CKD 4/6 inhibitor in combination with an endocrine agent has been shown to significantly prolong progression-free survival as compared with the endocrine agent alone in advanced breast cancer [24-26], and this effect 
was reported to be independent of the degree of endocrine resistance, hormone-receptor expression level, and PIK3CA mutational status [26].

In conclusion, although the study was terminated owing to the altered liver function, it showed there was a trend to greater shrinkage of tumor in the combination group. The combination of an AI with an oral fluoropyrimidine may become an attractive option as neoadjuvant therapy for ER-positive, HER2-negative breast cancer. A study is currently underway to evaluate the benefit of endocrine therapy combined with $\mathrm{S}-1$ as adjuvant (postoperative) therapy for ER-positive, HER2-negative breast cancer (POTENT study). The results of this ongoing study will further clarify the clinical role of adding metronomic chemotherapy to endocrine therapy for this phenotype of breast cancer.

Acknowledgements The authors would like to thank Drs. Masakazu Toi and Satoshi Morita, who acted as the members of the Independent Data Monitoring Board. Translational Research Informatics Center in Kobe served as the patient registration and clinical data center. Editorial support was provided by USACO Corporation. This work was supported by the Foundation for Biomedical Research and Innovation, Translational Research Informatics Center, under the funding contract with Taiho Pharmaceutical Co., Ltd., Japan.

Funding This work was supported by the Foundation for Biomedical Research and Innovation, Translational Research Informatics Center, under the funding contract with Taiho Pharmaceutical Co., Ltd., Japan.

\section{Compliance with ethical standards}

Conflict of interest Takahiro Nakayama has received honoraria from Chugai Pharmaceutical, AstraZeneca, Taiho Pharmaceutical, and Novartis Pharma. Norikazu Masuda has received honoraria from AstraZeneca and Chugai. Yasuo Miyoshi has received honoraria and research funding from AstraZeneca and Taiho. Shinzaburo Noguchi has received honoraria from AstraZeneca, Taiho, Chugai, Novartis, Takeda, and Daiichi-Sankyo, has received research funding from AstraZeneca, Taiho, Pfizer, Chugai, Eisai, Takeda, and Daiichi-Sankyo, and is an advisor for Taiho, AstraZeneca, and Novartis. The other authors declare that they have no potential conflicts of interest. Foundation for Biomedical Research and Innovation has received research grant from AstraZeneca.

Ethical standards The study was conducted in accordance with the ethical standards established in the 1964 Declaration of Helsinki and its later amendments. The study was approved by the institutional review board of each study site, and all patients participating in the study provided informed consent before enrollment.

Open Access This article is distributed under the terms of the Creative Commons Attribution 4.0 International License (http://creativeco mmons.org/licenses/by/4.0/), which permits unrestricted use, distribution, and reproduction in any medium, provided you give appropriate credit to the original author(s) and the source, provide a link to the Creative Commons license, and indicate if changes were made.

\section{References}

1. Baum M, Budzar AU, Cuzick et al (2002) Anastrozole alone or in combination with tamoxifen versus tamoxifen alone for adjuvant treatment of postmenopausal women with early breast cancer: first results of the ATAC randomised trial. Lancet 22:359(9324):2131-2139

2. Thürlimann B, Keshaviah A, Coates AS, Breast International Group (BIG) 198 Collaborative Group et al. (2005) A comparison of letrozole and tamoxifen in postmenopausal women with early breast cancer. N Engl J Med 353(26):27472757

3. Early Breast Cancer Trialists' Collaborative Group (EBCTCG) (2005) Effects of chemotherapy and hormonal therapy for early breast cancer on recurrence and 15year survival: an overview of the randomised trials. Lancet 365(9472):1687-1717

4. Wolmark N, Wang J, Mamounas E et al (2001) Preoperative chemotherapy in patients with operable breast cancer: nine year results from National Surgical Adjuvant Breast and Bowel Project B18. J Natl Cancer Inst Monogr (30):96-102

5. Andre F, Mazouni C, Liedtke C et al (2008) HER2 expression and efficacy of preoperative paclitaxel/FAC chemotherapy in breast cancer. Breast Cancer Res Treat 108(2): 183190

6. Toi M, Nakamura S, Kuroi K, Japan Breast Cancer Research Group (JBCRG) et al (2008) Phase II study of preoperative sequential FEC and docetaxel predicts of pathological response and disease free survival. Breast Cancer Res Treat 110(3):531539

7. Goldhirsch A, Ingle JN, Gelber RD, Panel members et al. (2009) Thresholds for therapies: highlights of the St Gallen International Expert Consensus on the primary therapy of early breast cancer 2009. Ann Oncol 20(8):13191329

8. Alba E, Calvo L, Albanell J et al (2012) Chemotherapy (CT) and hormonotherapy (HT) as neoadjuvant treatment in luminal breast cancer patients: results from the GEICAM/200603, a multicenter, randomized, phaseII study. Ann Oncol 23(12):30693074

9. Browder T, Butterfield CE, Kräling BM et al (2000) Antiangiogenic scheduling of chemotherapy improves efficacy against experimental drugresistant cancer. Cancer Res 60(7):18781886

10. Kerbel RS, Kamen BA (2004) The antiangiogenic basis of metronomic chemotherapy. Nat Rev Cancer 4(6):423736

11. Nukatsuka M, Saito H, Nakagawa F et al (2011) Oral fluoropyrimidine may augment the efficacy of aromatase inhibitor via the downregulation of estrogen receptor in estrogenresponsive breast cancer xenografts. Breast Cancer Res Treat 128(2):381390

12. Smith IE, Dowsett M, Ebbs SR, IMPACT Trialists Group et al. (2005) Neoadjuvant treatment of postmenopausal breast cancer with anastrozole, tamoxifen, or both in combination: the Immediate Preoperative Anastrozole, Tamoxifen, or Combined with Tamoxifen (IMPACT) multicenter doubleblind randomized trial. $\mathrm{J}$ Clin Oncol 23(22):5108-5116

13. Cataliotti L, Buzdar AU, Noguchi S et al (2006) Comparison of anastrozole versus tamoxifen as preoperative therapy in postmenopausal women with hormone receptorpositive breast cancer: the PreOperative Arimidex Compared to Tamoxifen (PROACT) trial. Cancer 106(10):2095-2103

14. Albain KS, Barlow WE, Ravdin PM, Breast Cancer Intergroup of North America et al. (2009) Adjuvant chemotherapy and timing of tamoxifen in postmenopausal patients with endocrine-responsive, nodepositive breast cancer: a phase 3, openlabel, randomised controlled trial. Lancet 374(9707):2055-2063

15. Noguchi S, Koyama H, Uchino J et al (2005) Postoperative adjuvant therapy with tamoxifen, tegafur plus uracil, or both in women with node-negative breast cancer: a pooled analysis of six randomized controlled trials. J Clin Oncol 23(10):2172-2184

16. Watanabe T, Sano M, Takashima $S$ et al (2002) Oral uracil and tegafur compared with classic cyclophosphamide, methotrexate, 
fluorouracil as postoperative chemotherapy in patients with nodenegative, high-risk breast cancer: National Surgical Adjuvant Study for Breast Cancer 01 Trial. J Clin Oncol 27(9):1368-1674

17. Park Y, Okamura K, Mitsuyama $S$ et al (2009) Uracil-tegafur and tamoxifen vs cyclophosphamide, methotrexate, fluorouracil, and tamoxifen in post-operative adjuvant therapy for stage I, II, or IIIA lymph node-positive breast cancer: a comparative study. $\mathrm{Br}$ J Cancer 101(4):598-604

18. Ohashi Y, Watanabe T, Sano M et al (2010) Efficacy of oral tegafururacil (UFT) as adjuvant therapy as compared with classical cyclophosphamide, methotrexate, and 5-fluorouracil (CMF) in early breast cancer: a pooled analysis of two randomized controlled trials (N.SASBC 01 trial and CUBC trial). Breast Cancer Res Treat 119(3):633-641

19. Taguchi T, Noguchi S (2009) Safety and compliance with UFT (tegafur and uracil) alone and in combination with hormone therapy in patients with breast cancer. Jpn J Cancer Chemother 36(9): 1465-1474

20. Toi M, Nakamura S, Kuroi K, Japan Breast Cancer Research Group (JBCRG) et al (2008) Phase II study of preoperative sequential FEC and docetaxel predicts of pathological response and disease free survival. Breast Cancer Res Treat 110(3):531-539

21. Llombart-Cussac A, Guerrero Á, Galán A et al (2012) Phase II trial with letrozole to maximum response as primary systemic therapy in postmenopausal patients with ER/PgR[+] operable breast cancer. Clin Transl Oncol 14(2):125-131
22. Allevi G, Strina C, Andreis D et al (2013) Increased pathological complete response rate after a longterm neoadjuvant letrozole treatment in postmenopausal oestrogen and/or progesterone receptor-positive breast cancer. Br J Cancer 108(8):1587-1592

23. Sakai T, Iwata H, Hasegawa Y et al (2014) First report of clinicopathological analysis in neoadjuvant treatment phase in NEOS:A randomized study of adjuvant endocrine therapy with or without chemotherapy for postmenopausal breast cancer patients who responded to neoadjuvant letrozole. San Antonio Breast Cancer Symposium P1-14-1

24. Finn RS, Crown JP, Lang I et al (2015) The cyclindependent kinase 4/6 inhibitor palbociclib in combination with letrozole versus letrozole alone as firstline treatment of oestrogen receptorpositive, HER2negative, advanced breast cancer (PALOMA1/ TRIO18): a randomised phase 2 study. Lancet Oncol 16(1):25-35

25. Finn RS, Martin M, Rugo HS et al (2016) Palbociclib and letrozole in advanced breast cancer. N Engl J Med 375(20):1925-1936

26. Cristofanilli M, Turner NC, Bondarenko I et al (2016) Fulvestrant plus palbociclib versus fulvestrant plus placebo for treatment of hormone-receptor-positive, HER2-negative metastatic breast cancer that progressed on previous endocrine therapy (PALOMA-3): final analysis of the multicentre, double-blind, phase 3 randomised controlled trial. Lancet Oncol 17(1):425-439 